\title{
VARIACIÓN DEL ESTADO NUTRICIONAL DURANTE EL TRATAMIENTO ANTITUBERCULOSO EN BENEFICIARIOS DEL PROGRAMA PANTBC
}

\author{
Juan Pablo Aparco ${ }^{1, a}$, Lucio Huamán-Espino ${ }^{1, b}$, Eddy R. Segura²,c
}

\begin{abstract}
RESUMEN
Objetivos. Analizar la variación nutricional de los beneficiarios actuales y pasados del Programa de Alimentación y Nutrición al Paciente Ambulatorio con Tuberculosis y Familia (PANTBC). Materiales y métodos. Se realizó un análisis secundario a partir de la valoración antropométrica por medio del índice de masa corporal (IMC) en beneficiarios actuales y pasados del programa PANTBC. Las mediciones se realizaron al inicio, segundo y quinto o sexto mes y se comparó la distribución del estado nutricional al inicio respecto al final (prueba de McNemar-Bowker). Resultados. Se incluyó información de 409 beneficiarios actuales y 110 beneficiarios pasados. Tanto en beneficiarios actuales como pasados se observó disminución progresiva de la condición de bajo peso $(\mathrm{IMC}<18,5)$ así como el aumento de la condición de sobrepeso. Los resultados de la tercera medición mostraron que el $57,7 \%$ de los beneficiarios actuales que iniciaron con bajo peso terminaron con peso normal, mientras el $20,7 \%$ de los que iniciaron con peso normal terminaron con sobrepeso. La comparación del estado nutricional al inicio respecto al final mostró cambios en la distribución $(p<0,05)$. Conclusiones. A lo largo del programa PANTBC existe variación del estado nutricional de los beneficiarios, con la tendencia de pasar de un nivel inferior a otro superior de IMC.
\end{abstract}

Palabras clave: Tuberculosis; Estado nutricional; Índice de masa corporal (fuente: DeCS BIREME).

\section{CHANGE IN NUTRITIONAL STATUS OVER THE COURSE OF ANTITUBERCULOSIS TREATMENT IN CURRENT AND PAST BENEFICIARIES OF THE PROGRAM PANTBC}

\begin{abstract}
Objectives. Analyze the nutritional changes among current and old beneficiaries of the Food and Nutrition Program for Outpatients with Tuberculosis and their Family (PANTBC, Spanish acronym). Materials and methods. A secondary analysis was conducted based on the anthropometric measurements with the body mass index (BMI) in current and old beneficiaries of the PANTBC program. The measurements were taken at the baseline, second and fifth or sixth month, and the distribution of the nutritional status was analyzed at baseline as compared to the endpoint (McNemar-Bowker test). Results. Information about 409 current beneficiaries and 110 old beneficiaries were included. A progressive decline in low weight as well as an increase in overweight were observed in both current and old beneficiaries (IMC<18.5). The results of the third measurement showed that $57.7 \%$ of current beneficiaries who started with low weight ended up with normal weight, while $20.7 \%$ of those who started with normal weight ended up with overweight. The analysis of the nutritional status at baseline compared to the endpoint showed changes in distribution $(p<0.05)$. Conclusions. Across the PANTBC program, there is a variation of beneficiaries' nutritional status, showing a tendency to go from a lower level to a higher level of BMI.
\end{abstract}

Key words: Tuberculosis; Nutritional status; Body mass index (source: MeSH NLM).

\section{INTRODUCCIÓN}

La tuberculosis (TB) es una de las principales enfermedades infectocontagiosas que afecta a los países en vías de desarrollo ${ }^{(1)}$. En el Perú, se estima que la morbilidad por esta enfermedad alcanzó la tasa de 129 por 100000 habitantes en el 2005 (2). Asimismo, se conoce que la TB afecta gravemente el estado nutricional debido al incremento del metabolismo inherente a la infección, así como a una reducción de

\footnotetext{
Centro Nacional de Alimentación y Nutrición, Instituto Nacional de Salud. Lima, Perú.

Facultad de Ciencias de la Salud, Universidad Peruana de Ciencias Aplicadas. Lima, Perú.

Nutricionista; ${ }^{\mathrm{b}}$ sociólogo; ${ }^{\mathrm{c}}$ médico magíster en Salud Pública y Epidemiología

Recibido: 31-05-12 Aprobado: 08-08-12
}

Citar como: Aparco JP, Huamán-Espino L, Segura ER. Variación del estado nutricional durante el tratamiento antituberculoso en beneficiarios del programa PANTBC. Rev Peru Med Exp Salud Publica. 2012;29(3):324-8. 
la ingesta calórica que finalmente afecta las reservas del organismo. Dentro de este contexto, es lógica y razonable la provisión de un complemento nutricional.

En Perú, el tratamiento farmacológico se complementa con medidas de soporte nutricional. El Programa de Alimentación y Nutrición al Paciente Ambulatorio con Tuberculosis y Familia (PANTBC) es una de las intervenciones del Ministerio de Salud para brindar apoyo nutricional a los pacientes que están recibiendo tratamiento. El objetivo principal del programa es contribuir a la recuperación integral del paciente ambulatorio afectado de TB, y a la protección de su familia mediante actividades de educación, evaluación nutricional y complementación alimentaria con una canasta de alimentos que brinde el $28 \%$ de los requerimientos calóricos y el $38 \%$ de los requerimientos proteicos de la familia (un paciente y dos contactos) ${ }^{(3,4)}$. La experiencia de proveer soporte nutricional para esta enfermedad se ha dado en varias regiones a nivel mundial y aunque sus beneficios no son del todo claros, puede servir como incentivo para promover la adherencia al tratamiento médico ${ }^{(5)}$.

En nuestro medio se han realizado investigaciones acerca del estado nutricional en el curso de la enfermedad y el tratamiento de TB ${ }^{(6-9)}$; sin embargo, existe limitada información sobre la evolución del índice de masa corporal (IMC) a lo largo del programa PANTBC ${ }^{(10)}$. El objetivo de la presente investigación es evaluar el cambio en el estado nutricional, estimado a través del IMC, en beneficiarios actuales y pasados de este programa.

\section{MATERIALES Y MÉTODOS}

Se realizó un análisis secundario a partir del estudio para evaluar el programa PANTBC 2010 a nivel nacional, ejecutado por una institución contratada por el Instituto Nacional de Salud mediante un mecanismo de consultoría. Este estudio fue de tipo descriptivo transversal, a modo de línea de base. Para ello se utilizó el modelo de enfoque sistémico, donde los insumos y procesos se valoraron en base al análisis de la gestión del PANTBC a cargo del Programa Nacional de Asistencia Alimentaria (PRONAA) en Lima y de los municipios provinciales en el resto del país. Los efectos se midieron a través del aporte nutricional en cantidad y calidad de la ración distribuida y los conocimientos, actitudes y prácticas (CAP) de los responsables del programa en los establecimientos de salud. Los resultados se midieron en base a los cambios observados en el estado nutricional de los beneficiarios actuales y pasados ${ }^{(4)}$. El estudio fue aprobado por el Comité de Ética de la Asociación Benéfica PRISMA.
Para la presente investigación nutricional se utilizó la información antropométrica básica (peso y talla) a partir de las historias clínicas de los beneficiarios pasados (quienes ya habían concluido el tratamiento y el programa al momento de ejecutar el estudio) y de los beneficiarios actuales (quienes aún recibían tratamiento antituberculoso y el apoyo del PANTBC al momento del estudio). Solo se consideró el registro de pacientes para quienes consistentemente existieron datos antropométricos en los puntos del tiempo requerido.

La investigación inicial siguió un muestreo de tipo probabilístico, polietápico, independiente por cada dominio de estudio y con probabilidades proporcionales al tamaño. Fue realizado a nivel nacional y con inferencia en dos dominios (Lima y resto municipal). Los datos incluidos en la presente investigación provienen de ambos dominios.

La determinación del estado nutricional se hizo a través del índice de masa corporal (IMC), documentándose en tres momentos. Para beneficiarios actuales: al inicio, a los dos y cinco meses del tratamiento, y para beneficiarios pasados: al inicio, a los dos y seis meses del tratamiento. De las seis mediciones, solo la del quinto mes en el grupo de beneficiarios actuales se llevó a cabo por medio del equipo de campo del estudio original. El resto de mediciones se recogieron de los registros del establecimiento de salud.

Se utilizó estadística descriptiva para las descripciones univariadas y los cruces de variables. Se describió frecuencias absolutas (número) y relativas (porcentajes) de la distribución del estado nutricional según categorías del IMC (bajo peso: IMC<18,5; normal: IMC 18,5 a 24,9; sobrepeso: IMC 25,0 a 29,9 y obesidad: IMC $\geq 30,0$ ) en cada punto del tiempo (al inicio, a los dos meses y a los cinco o seis meses). Para comparar la distribución de las categorías del estado nutricional al inicio del estudio respecto al final, se empleó la prueba de simetría de McNemar-Bowker puesto que se trataban de medidas correlacionadas (pareadas por sujeto), y para ver los cambios en los promedios del IMC se empleó la prueba de ANOVA para promedios correlacionados, luego se procedió a comparaciones de pares de promedios específicos tomando en cuenta el ajuste de Bonferroni. Se consideró como significativos los valores $p<0,05$. El análisis de datos se hizo en el programa SPSS V.19.0 (SPSS Inc. Chicago, IL).

\section{RESULTADOS}

Se encuestaron en total 710 beneficiarios actuales del programa PANTBC. Para efectos de la valoración 
Tabla 1. Características generales de los beneficiarios actuales y pasados del programa PANTBC elegibles para la valoración de la progresión del estado nutricional. Perú, 2010.

\begin{tabular}{|c|c|c|}
\hline Características & $\begin{array}{c}\text { Beneficiarios } \\
\text { actuales } \\
\text { N. }{ }^{\circ}(\%)\end{array}$ & $\begin{array}{c}\text { Beneficiarios } \\
\text { pasados } \\
\text { N. }{ }^{\circ}(\%)\end{array}$ \\
\hline Edad promedio (DE) & $34(17,5)$ & $33,2(15,3)$ \\
\hline \multicolumn{3}{|l|}{ Sexo } \\
\hline Femenino & $155(37,9)$ & $42(38,2)$ \\
\hline Masculino & $254(62,1)$ & $68(61,8)$ \\
\hline \multicolumn{3}{|l|}{ Raciones PANTBC recibidas } \\
\hline Primera ración completa & $279(68,1)$ & $98(88,8)$ \\
\hline Segunda ración completa & $286(69,9)$ & $99(89,9)$ \\
\hline Tercera ración completa & $285(69,7)$ & $90(81,8)$ \\
\hline Cuarta ración completa & $337(82,4)$ & $94(85,5)$ \\
\hline Quinta ración completa & $342(83,7)$ & $100(90,6)$ \\
\hline \multicolumn{3}{|l|}{ Dominio geográfico } \\
\hline Lima & $205(50,1)$ & $66(59,7)$ \\
\hline Resto Municipal & $204(49,9)$ & $44(40,3)$ \\
\hline Total & $409(100)$ & $110(100)$ \\
\hline
\end{tabular}

DE: desviación estándar.

nutricional se pudo obtener datos seriados (para los tres puntos del tiempo) de 409 beneficiarios actuales del programa y de 110 beneficiarios pasados. La edad de los beneficiarios actuales del programa PANTBC osciló entre 11 y 88 años, con una edad promedio de $34 \pm 17,5$ años; el $62,1 \%$ (254/409) eran varones y el $50,8 \%(208 / 409)$ eran solteros. Con relación a la condición laboral, 40,9\% (167/409) manifestó que trabajaba; respecto al seguro de salud, 63,1\% (258/409) de los beneficiarios actuales del programa cuenta con algún tipo de seguro de salud. El resto de características de los beneficiarios actuales y pasados se muestran en la Tabla 1.

Tabla 2. Distribución de las categorías del IMC en cada una de las tres mediciones en beneficiarios actuales y pasados del Programa PANTBC 2010.

\begin{tabular}{|c|c|c|c|}
\hline $\begin{array}{l}\text { Categoría de } \\
\text { IMC }\end{array}$ & $\begin{array}{c}\text { Primera } \\
\text { medición } \\
\mathrm{N} .{ }^{\circ}(\%)\end{array}$ & $\begin{array}{c}\text { Segunda } \\
\text { medición } \\
\text { N. }{ }^{\circ}(\%)\end{array}$ & $\begin{array}{c}\text { Tercera } \\
\text { medición } \\
\mathbf{N} .^{\circ}(\%)\end{array}$ \\
\hline \multicolumn{4}{|c|}{ Beneficiarios actuales $\left(\mathrm{N}^{\circ}{ }^{\circ}=409\right)$} \\
\hline Bajo peso & $78(19,1)$ & $63(15,4)$ & $39(9,5)$ \\
\hline Normal & $271(66,3)$ & $258(63,1)$ & $254(62,1)$ \\
\hline Sobrepeso & $55(13,4)$ & $74(18,1)$ & $99(24,2)$ \\
\hline Obesidad & $5(1,2)$ & $14(3,4)$ & $17(4,2)$ \\
\hline \multicolumn{4}{|c|}{ Beneficiarios pasados $\left(\mathrm{N}^{\circ}=110\right)$} \\
\hline Bajo peso & $19(17,3)$ & $14(12,7)$ & $8(7,3)$ \\
\hline Normal & $81(73,6)$ & $77(70,0)$ & $69(62,7)$ \\
\hline Sobrepeso & $10(9,1)$ & $18(16,4)$ & $28(25,5)$ \\
\hline Obesidad & $0(0)$ & $1(0,9)$ & $5(4,5)$ \\
\hline
\end{tabular}

Mediciones: primera: al inicio; segunda: al segundo mes; tercera: para beneficiarios actuales al quinto mes, para beneficiarios pasados al sexto mes.
Tabla 3. Distribución de las categorías de IMC en la tercera medición de acuerdo al IMC en la primera medición en beneficiarios actuales y pasados del Programa PANTBC 2010.

\begin{tabular}{|c|c|c|c|}
\hline \multirow[b]{2}{*}{$\begin{array}{l}\text { IMC al ingreso } \\
\text { al programa }\end{array}$} & \multicolumn{3}{|c|}{$\begin{array}{c}\text { Distribución del estado nutricional en } \\
\text { la tercera medición }\end{array}$} \\
\hline & $\begin{array}{c}\text { Bajo peso } \\
\text { N. }{ }^{\circ}(\%)\end{array}$ & $\begin{array}{l}\text { Normal } \\
\text { N. }{ }^{\circ}(\%)\end{array}$ & $\begin{array}{c}\text { Sobrepeso- } \\
\text { Obesidad } \\
\text { N. }{ }^{\circ}(\%)\end{array}$ \\
\hline \multicolumn{4}{|c|}{ Beneficiarios actuales $\left(\mathrm{N}^{\circ}{ }^{\circ}=409\right)$} \\
\hline Bajo peso & $32(41,0)$ & $45(57,7)$ & $1(1,3)$ \\
\hline Normal & $6(2,2)$ & $205(75,6)$ & $60(22.1)$ \\
\hline $\begin{array}{l}\text { Sobrepeso- } \\
\text { Obesidad }\end{array}$ & $1(1,7)$ & $4(6.7)$ & $55(91.7)$ \\
\hline \multicolumn{4}{|c|}{ Beneficiarios pasados $\left(\mathrm{N} .{ }^{\circ}=110\right)$} \\
\hline Bajo peso & $7(36,8)$ & $12(63,2)$ & $0(0,0)$ \\
\hline Normal & $1(1,2)$ & $55(67,9)$ & $25(30.8)$ \\
\hline $\begin{array}{l}\text { Sobrepeso- } \\
\text { Obesidad }\end{array}$ & $0(0,0)$ & $2(20,0)$ & $8(80,0)$ \\
\hline
\end{tabular}

En la Tabla 2 se aprecia la distribución de las categorías del IMC de los sujetos en cada una de las tres mediciones del programa PANTBC, tanto en beneficiarios actuales como en pasados. En la Tabla 3 se muestra la distribución al final del estudio en beneficiarios actuales y pasados (cinco y seis meses respectivamente de haber ingresado al programa PANTBC) y la distribución que tenían al inicio. Se aprecia la redistribución de sujetos desde categorías inferiores de IMC hacia superiores, tanto en beneficiarios actuales como pasados.

En el caso de los beneficiarios actuales, la distribución de categorías del estado nutricional al inicio del estudio, mostró cambios significativos respecto a la distribución al final (prueba de McNemar-Bowker, $p<0,05$ ), con una tendencia al incremento del promedio del IMC (ANOVA para promedios repetidos $p<0,05$ ). Así, en la primera medición el promedio de IMC fue de $21,7 \pm 3,5$, en la segunda fue $22,2 \pm 3,7$; y en la tercera medición fue $23,12 \pm 3,78$, estas diferencias persistieron luego de comparaciones de pares de medias específicas tomando en cuenta el ajuste de Bonferroni.

\section{DISCUSIÓN}

Según los hallazgos de esta investigación, existe un cambio en la distribución del estado nutricional en beneficiarios actuales a lo largo del curso del tratamiento antituberculoso en el contexto del Programa PANTBC, los cuales son consistentes con los resultados encontrados para los beneficiarios pasados. Asimismo, se pudo evidenciar un aumento significativo del IMC de los beneficiarios actuales en los tres momentos de 
valoración del estado nutricional, lo cual se evidencia en una migración de sujetos entre categorías del IMC desde el inicio del programa hasta casi el final, con tendencia a favorecer la migración hacia una categoría superior, encontrándose mayor cambio en la categoría de bajo peso a normal, seguido por normal a sobrepeso u obesidad.

Diversas razones podrían explicar el aumento de peso durante el tratamiento antituberculoso, entre ellas, el aumento del apetito e ingesta de alimentos, la reducción de las demandas de energía y la mejora de la eficiencia metabólica entre otras (11,12); aunque la ganancia de peso está limitada al aumento de tejido graso ${ }^{(13,14)}$. Sin embargo, también se debe tomar en cuenta que en los beneficiarios del PANTBC está inherente el aporte nutricional de la canasta alimentaria. En ese sentido, lo ideal sería realizar una valoración de las gradientes de aporte nutricional, la evaluación del cambio del IMC (y otras mediciones que permitan medir los compartimentos corporales) y tomar en cuenta otras potenciales variables que no han sido colectadas en el estudio inicial (como son el tipo de tuberculosis según resistencia, dilución del aporte de la canasta en el hogar, nivel de actividad física durante la enfermedad y adherencia al tratamiento).

Existe limitada información sobre el apoyo nutricional durante el tratamiento de TB. Dos ensayos clínicos encontraron que los pacientes en tratamiento antituberculoso que recibieron suplemento nutricional tuvieron mayor aumento de peso corporal en comparación con un grupo control, y que al final del estudio el grupo suplementado tuvo más cantidad de tejido magro ${ }^{(15,16)}$. Otro estudio en Inglaterra también encontró recuperación nutricional en pacientes con tratamiento de TB; sin embargo, a los 12 meses los niveles de albúmina sérica se mantenían bajos ${ }^{(17)}$.

En nuestro país también se han realizado estudios que relacionan el estado nutricional y los resultados del tratamiento de TB. Entre los hallazgos más relevantes tenemos que un IMC $<18$ al inicio del tratamiento está asociado con un mayor riesgo de mortalidad ${ }^{(6)}$. Otros estudios encontraron que la evolución del peso a lo largo del tratamiento era pronóstico para la enfermedad $(7,8)$. Chung et al. encontraron que la presencia de sobrepeso y obesidad estaba asociada con el desarrollo de efectos adversos durante la terapia antituberculosa ${ }^{(9)}$. Al respecto, es preciso mencionar que algunos autores han señalado que la diabetes y la obesidad (entre otros trastornos metabólicos que suprimen al sistema inmunológico) propician la reactivación de la infección (18). Asimismo, otro estudio ha sugerido que el tejido adiposo puede constituir un vasto reservorio donde el bacilo puede persistir por largos periodos y evadir la acción de los medicamentos y del sistema inmune ${ }^{(19)}$.

La evaluación de impacto del programa PANTBC realizada por PRISMA encontró reducción de la proporción de beneficiarios con bajo peso, poca variación en la categoría normal y aumento en sobrepeso; sin embargo, no se consideró en el análisis la condición nutricional inicial de los beneficiarios (20). Asimismo, Salazar et al. evaluaron la variación del estado nutricional en beneficiarios de PANTBC de Arequipa en forma retrospectiva a partir de registros de 1996 al 2002 y encontraron que a lo largo del tratamiento el porcentaje de sujetos con bajo peso disminuyó (de 25 a $14 \%$ ) mientras que el del peso normal aumentó (de 64 a $84 \%$ ); en tanto que el sobrepeso se redujo en $100 \%$ al final de la intervención ${ }^{(10)}$. Este estudio no evaluó el cambio del IMC en las tres valoraciones antropométricas realizadas, se basó en registros retrospectivos y fue circunscrito a la zona de Arequipa metropolitana.

Los resultados de esta investigación también deben llamar a la reflexión sobre la forma y el destino del complemento nutricional. Actualmente, todos los sujetos con diagnóstico de TB son elegibles para recibir la canasta alimentaria independientemente de su estado nutricional y composición familiar al momento de ingreso al programa. En aras de maximizar los resultados y hacer más efectivo el programa, se debería tomarse en cuenta el estado nutricional al ingreso al programa para priorizar mayor aporte de energía en aquellos beneficiarios que ingresan al programa con bajo peso o peso normal, en tanto los que ingresan con sobrepeso u obesidad podrían recibir una canasta alimentaria con diferente objetivo nutricional al de proveer mayor energía.

Nuestro estudio presenta algunas limitaciones: 1) La heterogeneidad en la medición del IMC, ya que la mayoría de mediciones se obtuvieron a partir de registros recogidos para otro propósito, 2) No contar con otras covariables de interés y relevantes para un análisis más profundo y 3) No es posible una comparación directa contra un grupo control dado por aquellos que no reciben PANTBC pues, por condición básica del programa, todos son elegibles, y aunque en diferentes medidas y raciones, todos reciben canasta de alimentos. A pesar de lo mencionado, el estudio tiene una gran fortaleza que es la evaluación prospectiva y no solo transversal, del estado nutricional. De manera adicional, se pudo aprovechar la información disponible para evaluar el cambio del estado nutricional y del IMC en beneficiarios según condición de ingreso, lo cual no se había analizado en las evaluaciones previas del programa PANTBC ${ }^{(4,20)}$. 
En conclusión, los resultados del estudio muestran que existe cambio del estado nutricional y un aumento significativo del promedio del IMC en beneficiarios actuales a lo largo del curso del tratamiento antituberculoso en el contexto del programa PANTBC; sin embargo, esta variación debería ser evaluada con una metodología más rigurosa, en forma homogénea y con la consideración de otras variables relevantes, como las discutidas previamente, para determinar la contribución o no del PANTBC en el incremento del peso. A nivel de programa, los hallazgos informan sobre qué grupos podrían ser priorizados con la finalidad de maximizar los resultados, en el plano nutricional, sobre todo cuando el beneficio de incentivos nutricionales sobre la adherencia o el estado nutricional no es del todo claro ${ }^{(5)}$

Contribuciones de autoría: JPA y LHE participaron en el diseño del estudio, supervisión de la recolección y procesamiento y análisis de datos. ERS realizó el análisis de datos y escribió el borrador del artículo. Todos los autores revisaron críticamente el manuscrito y aprobaron la versión a publicar.

Fuentes de financiamiento: autofinanciado.

Conflictos de interés: los autores declaran no tener conflictos de interés en la publicación de este artículo.

\section{REFERENCIAS BIBLIOGRÁFICAS}

1. World Health Organization (WHO). Global tuberculosis control 2009 . Epidemiology, strategy, financing. WHO Report 2009. Ginebra: WHO; 2009.

2. Perú, Ministerio de Salud (MINSA). Plan Estratégico Multisectorial de la Respuesta Nacional a la Tuberculosis en el Perú 2010-2019. Lima: MINSA; 2010.

3. Perú, Ministerio de Desarrollo e Inclusión Social, Programa Nacional de Asistencia Alimentaria (PRONAA). Conociendo el PANTBC: Un programa de apoyo a la recuperación de personas afectadas por la TBC. Lima: PRONAA; 2005.

4. Instituto Cuanto. Informe Final: Evaluación del Programa PANTBC 2010. Lima: Instituto Cuanto; 2010.

5. Sinclair D, Abba K, Grobler L, Sudarsanam TD. Nutritional supplements for people being treated for active tuberculosis. Cochrane Database Syst Rev. 2011;(11):CD006086.

6. Bernabé-Ortiz A. Factores asociados a supervivencia en pacientes con tuberculosis en Lima, Perú. Rev Chil Infec. 2008;25(2):104-7.

7. Bernabe-Ortiz A, Carcamo CP, Sanchez JF, Rios J. Weight variation over time and its association with tuberculosis treatment outcome: a longitudinal analysis. PloS One. 2011;6(4):e18474.

8. Krapp F, Véliz JC, Cornejo E, Gotuzzo E, Seas C. Bodyweight gain to predict treatment outcome in patients with pulmonary tuberculosis in Peru. Int J Tuberc Lung Dis 2008;12(10):1153-9.

9. Chung-Delgado K, Revilla-Montag A, Guillen-Bravo S, Velez-Segovia E, Soria-Montoya A, Nuñez-Garbin A, et al. Factors associated with antituberculosis medication adverse effects: a case-control study in Lima, Peru. PloS One. 2011;6(11):e27610.

10. Salazar V, Figueroa R, Machaca R. Variación del estado nutricional de los beneficiarios del Programa PANTBC en Arequipa Metropolitana 19962002. Rev Peru Med Exp Salud Publica. 2005;22(2):134-8.

11. United States Agency for International Development (USAID). Nutrition and Tuberculosis: A review of the literature and considerations for TB control programs. Washington, DC: USAID; 2008.

12. Vasanta M, Gopi PG, Subramani R. Weightgain in patientswith tuberculosis treated under directly observed treatment short-course (DOTS). Indian J Tubrc. 2009;56(1):5-9.

13. Macallan DC. Malnutrition in tuberculosis. Diagn Microbiol Infect Dis. 1999;34(2):153-7.

14. Schwenk A, Hodgson L, Wright A, Ward LC, Rayner CF, Grubnic S, et al. Nutrient partitioning during treatment of tuberculosis: gain in body fat mass but not in protein mass. Am J Clin Nutr. 2004;79(6):006-12.

15. Paton NI, Chua YK, Earnest A, Chee CB. Randomized controlled trial of nutritional supplementation in patients with newly diagnosed tuberculosis and wasting. Am J Clin Nutr. 2004;80(2):460-5.

16. Jahnavi G, Sudha CH. Randomised controlled trial of food supplements in patients with newly diagnosed tuberculosis and wasting. Singapore Med J. 2010;51(12):957-62.

17. Onwubalili JK. Malnutrition among tuberculosis patients in Harrow, England. Eur J Clin Nutr. 1988;42(4):363-6.

18. Barrios-Payán JA, Castañón-Arreola M, Flores-Valdez MA, HernándezPando R. Aspectos biológicos, clínicos y epidemiológicos de la tuberculosis latente. Salud Publica Mex. 2010;52:70-8.

19. Neyrolles O, Hernández-Pando R, Pietri-Rouxel F, Fornès P, Tailleux $\mathrm{L}$, et al. Is adipose tissue a place for Mycobacterium tuberculosis persistence? PLoS One. 2006; 1:e43.

20. Asociación Benéfica Prisma. Informe Final: Evaluación de Impacto Nutricional para el PANTBC 2002. Lima: Asociación Benéfica Prisma; 2002.

Correspondencia: Juan Pablo Aparco

Dirección: Jr. Zorritos 1399 block 55 dpto

501, Lima 1, Perú

Teléfono: (51) 992922315

Correoelectrónico:japarco@ins.gob.pe 